Pacific Journal of Mathematics

COVERING MANIFOLDS WITH CELLS 


\title{
COVERING MANIFOLDS WITH CELLS
}

\author{
R. P. Osborne and J. L. Stern
}

In attempting to triangulate a topological manifold, one would like to be able to cover a manifold with closed cells whose intersections are nice. This paper is a study of minimal coverings of manifolds by open cells and a method of improving the intersections as the connectivity allows. The principal theorem is the following.

Theorem 1: If $M^{n}$ is a $k$-connected topological $n$-manifold (without boundary) and $q$ is the minimum of $k$ and $n-3$, then $M^{n}$ can be covered by $p$ open cells if $p(q+1)>n$. Futhermore, these cells may be chosen so that the intersection of any collection of these cells is $(q-1)$-connected.

As a consequence of this theorem it is shown that a contractible open $n$-manifold $(n \geqq 5)$ is the union of two open cells whose intersection is a contractible open manifold. One might note for instance that a 3-connected 10-manifold can be covered by 3 open cells whose intersections are 2 -connected.

1. Definitions and notation. An n-manifold is a connected separable locally Euclidean metric space. Superscripts will denote dimension. If $K^{r}$ is an abstract simplicial complex, we denote its carrier by $\left|K^{r}\right|$. The $s$-skeleton of $K^{r}$ will be denoted by $K^{s}$. The complementary skeleton (sometimes called the dual skeleton) of $K^{s}$ is defined to be the union of all simplexes in the first barycentric subdivision of $K^{r}$ whose carriers do not intersect $\left|K^{s}\right|$. We denote the complementary skeleton of $K^{s}$ by $K_{*}^{s}$ and note that the dimension of $K_{*}^{s}$ is $r-s-1$. If $\Psi$ is a homeomorphism of the unit ball in $E^{n}$ into a manifold, then we denote by $|\Psi|$ the image of $\Psi$ and by $|\Psi|_{\alpha}$, $0 \leqq \alpha \leqq 1$ the image under $\Psi$ of the ball of radius $\alpha$.

2. Covering by cells. In what follows we shall rely heavily on the topological engulfing of Newman [3].

THEOREM 2.1. Let $X$ be a locally tame closed set of dimension $k \leqq n-3$ in a k-connected topological $n$-manifold $M^{n}$, and let $U$ be a $(k-1)$-connected open set in $M^{n}$ such that $X \sim U$ is compact. Then there is a homeomorphism $h: M^{n} \rightarrow \rightarrow M^{n}$ such that $X \subset h(U)$ and $h$ is the identity on the complement of a compact set in $M^{n}$.

Using this engulfing we shall prove

THEOREM 2.2. Let $M^{n}$ be a k-connected topological n-manifold 
and let $q$ be the minimum of $k$ and $n-3$, then $M^{n}$ can be covered by $p$ open $n$-cells if $p(q+1)>n$.

This theorem is proven by induction using the following lemmas.

Lemma 2.3. Let $K^{r}$ be a finite subcomplex of a triangulation $T$ of $E^{n}$, let $U, U^{\prime}$ and $U^{\prime \prime}$ be open sets in $E^{n}$ such that $U \subset U^{\prime} \subset U^{\prime \prime}$, no simplex in $|T|$ intersects both $U$ and $E^{n} \sim U^{\prime}$, no simplex in $|T|$ intersects both $U^{\prime}$ and $E^{n} \sim U^{\prime \prime}$, and $K^{s} \subset U^{\prime \prime}$. Suppose $V \subset E^{n}$ is an open set containing $\left|K_{*}^{s}\right|$. Then there is a homeomorphism $h$ : $E^{n} \rightarrow \longrightarrow E^{n}$ such that (i) $K^{r} \subset h\left(U^{\prime \prime}\right) \cup V$, (ii) $h \mid\left(U \cup\left|K^{s}\right| \cup\left|K_{*}^{s}\right|\right)=1$ and (iii) there is a compact set $A \subset E^{n}$ such that $h \mid E^{n} \sim A=1$.

Lemma 2.4. Let $M^{n}$ be a k-connected $n$-manifold and let $q$ be the minimum of $k$ and $n-3$. Let $|\phi|$ be a cell in $M^{n}, 0 \leqq \alpha \leqq 1$ and $\left|K^{r}\right|$ an $r$-dimensional polyhedron in $|\phi|$. Let $\left|\Psi_{1}\right|,\left|\Psi_{2}\right|, \cdots,\left|\Psi_{m}\right|$ be $n$-cells in $M^{n}$ where $m(q+1)>r$, then there exist homeomorphisms $h_{1}, h_{2}, \cdots, h_{m}$ of $M^{n}$ onto itself such that

$$
K^{r} \subset h_{1}\left(\left|\stackrel{\circ}{\Psi}_{1}\right|\right) \cup h_{2}\left|\stackrel{\circ}{\Psi}_{2}\right| \cup \cdots \cup h_{m}\left|\stackrel{\circ}{\Psi}_{m}\right|
$$

and $\left|\Psi_{1}\right|_{\alpha} \subset h_{i}\left(\left|\Psi_{i}\right|_{\Gamma(\alpha+1) / 2]}\right)$.

Sketch of the proof of Lemma 2.3. There exists a homeomorphism $g$ of $K^{r}$ onto itself such that $g\left(U^{\prime \prime} \cap\left|K^{r}\right|\right) \cup\left(V \cap\left|K^{r}\right|\right)=\left|K^{r}\right|$ and $g \mid\left(\left|K^{r}\right| \cap U^{\prime}\right)=1$. (To get $g$ push out linearly from $K_{*}^{s}$. See ([6], p. 570) for more details on this push.) We extend $g$ by coneing to get a homeomorphism $h$ of $E^{n}$ onto itself so that $h$ is the identity on the complement of the union of all $n$-simplexes in $E^{n}$ having a face in $\left|K^{r}\right|$ that does not lie in $U^{\prime} . \quad h$ is the desired homeomorphism.

Proof of Lemma 2.4. Let $T$ be a triangulation of $|\phi|$ such that $K^{r}$ is a subcomplex of $T$. We proceed by induction on $b=[r /(q+1)]$. If $b=0$ then $r \leqq q$. Applying the method of Connell [3] we stretch $\left|\Psi_{1}\right|_{1}$ over $\left|\Psi_{1}\right|_{[(\alpha+1) / 2]} \cup\left|K^{r}\right|$ keeping $\left|\Psi_{1}\right|_{\alpha}$ fixed. Assume now that the theorem is true for every $r^{\prime}$ such that $\left[r^{\prime} /(q+1)\right]<b$ and suppose $[r /(q+1)]=b$ and $m(q+1)>r$. Let $T_{1}$ be a subdivision of $T$ such that no simplex of $T_{1}$ meets $\mathrm{Bd}\left|\Psi_{m}\right|_{[(\alpha+1) / 2]}$ and $\mathrm{Bd}\left|\Psi_{m}\right|_{1}$ or $\mathrm{Bd}\left|\Psi_{m}\right|_{\alpha}$ and $\mathrm{Bd}\left|\Psi_{m}\right|_{[(\alpha+1) / 2]}$. Let $\left|K_{1}^{r}\right|$ be the complex $\left|K^{r}\right|$ after subdivision. Assume $\left|\Psi_{1}\right|,\left|\Psi_{2}\right|, \cdots,\left|\Psi_{m}\right|$ are given $n$-cells. As in the case $b=0$ we can stretch $\left|\Psi_{m}\right|_{1}$ by $h_{m}$ over $\left|\Psi_{m}\right|_{[(\alpha+1) / 2]} \cup\left|K_{1}^{q}\right|$ keeping $\left|\Psi_{m}\right|_{[(\alpha+1) / 2]}$ fixed.

By the inductive hypothesis there are homeomorphisms $h_{1}, h_{2}, \cdots$, $h_{m-1}$ such that $\left|K_{1}^{q}\right| \subset \bigcup_{i+1}^{m-1} h_{i}\left(\left|\Psi_{i}\right|\right)$ and $\left.h_{i}|| \Psi_{i}\right|_{[(\alpha+1) / 2]}=1, i=1,2, \cdots$, 
$m-1$. We apply Lemma 2.3 to $\left|K_{1}^{q}\right|$ with $U=\left|\stackrel{\circ}{\Psi}_{m}\right| \cap|\stackrel{\circ}{\phi}|, U^{\prime}=$ $\left|\stackrel{\circ}{\Psi}_{m}\right|_{\Gamma(\alpha+1) / 2]} \cap|\stackrel{\circ}{\phi}| . \quad U^{\prime \prime}=\left|\stackrel{\circ}{\Psi}_{m}\right|_{1} \cap|\stackrel{\circ}{\phi}|$ and $V=\bigcup_{i+1}^{m-1} h_{i}\left(\left|\Psi_{i}\right|_{1}\right)$, so get the desired homeomorphism $h_{m}$.

Proof of Theorem 2.2. Let $\left|\phi_{1}\right|,\left|\phi_{2}\right|, \cdots$ be a collection of cells in $M^{n}$ such that $\left|\phi_{1}\right|_{\alpha},\left|\phi_{2}\right|_{\alpha}, \cdots$ covers $M^{n}$ for some $\alpha, 0<\alpha<1$. (In case $M^{n}$ is compact, a finite sequence of cells would suffice.) Choose a sequence $\left(\alpha_{i}\right)$ of real numbers so that $\alpha<\alpha_{1}<\alpha_{2} \cdots<1$. Using Lemma 2.4 we stretch $\left|\phi_{1}\right|_{\alpha_{1}}\left|\phi_{2}\right|_{\alpha_{1}} \cdots\left|\phi_{p}\right|_{\alpha_{1}}$, over $\left|\phi_{p+1}\right|$ by $h_{1,1} \cdots h_{p, 1}$ so that $\left|\phi_{i}\right|_{\alpha} \subset h_{i, 1}\left(\left|\phi_{i}\right|_{\alpha_{1}}\right)$ for each $i=1,1, \cdots, p$. Next we stretch $\bigcup_{i=1}^{p} h_{i}\left(\left|\phi_{i}\right|_{\alpha_{1}}\right)$ over $\left|\phi_{p+2}\right|_{\alpha}$ by $h_{1,2}, h_{2,2}, \cdots, h_{p, 2}$ as before. Continuing indictively we get $\left|\phi_{i}\right|_{\alpha} \subset h_{i, 1}\left|\phi_{i}\right|_{\alpha_{1}} \subset h_{i, 2} h_{i, 1}\left(\left|\phi_{i}\right|_{\alpha_{2}}\right) \cdots$ which is a monotone union of open $n$-cells. By [1] this union is an open cell for each $i$. Thus we have covered $M^{n}$ by open cells.

Note that if $M^{n}$ were compact, it could be covered by $p$ closed cells with bicollared boundaries.

3. Improving the intersections of the covering cells. The following lemmas will enable us to improve the connectivity of the intersections of the covering cells. In referring to the homotopy groups we omit reference to the fixed base point even though we do not assume the sets to be path connected.

Lemma 3.1. If $A \subset E^{n}$ is compact, $U$ is a neighborhood of $A$ and $i_{*}: \Pi_{k}(A) \rightarrow \Pi_{k}(U)$ is the map induced by inclusion, then $i_{*}\left(\Pi_{k}(A)\right)$ is finitely generated.

Proof. Let $|K|$ be a finite polyhedron in $E^{n}$ such that $A \subset|K| \subset U$. Then $\Pi_{k}(|K|)$ is finitely generated for each $k$, so factoring the map $i_{*}$ through $\Pi_{k}(|K|)$ we see that $i_{*}\left(\Pi_{k}(A)\right)$ is finitely generated.

Lemma 3.2. Let $M^{n}$ be a $q$-connected $n$ manifold $(q \leqq n-3)$ and let $\left|\Psi_{1}\right|$ and $\left|\Psi_{2}\right|$ be $n$-cells in $M$ and let $0<\alpha<\beta<1$. Then there exists a homeomorphism $h: M^{n} \rightarrow M^{n}$ such that $\left.h|| \Psi_{1}\right|_{\alpha}=1$ and if $i:\left(\left|\Psi_{1}\right|_{\alpha} \cap\left|\Psi_{2}\right|_{\alpha}\right) \rightarrow h\left(\left|\Psi_{1}\right|_{\beta} \cap\left|\Psi_{2}\right|_{\beta}\right)$ is the inclusion then

$$
i_{*}: I I_{k}\left(\left|\Psi_{1}\right|_{\alpha} \cap\left|\Psi_{2}\right|_{\alpha}\right) \rightarrow \Pi_{k}\left(h\left(\left|\Psi_{1}\right|_{\beta} \cap\left|\Psi_{2}\right|_{\beta}\right)\right)
$$

is trivial for $k=1,2, \cdots, q-1$.

Proof. Note that $\left|\stackrel{\circ}{\Psi}_{1}\right|_{[(\alpha+\beta) / 2]} \cap\left|\stackrel{\circ}{\Psi}_{2}\right|_{[(\alpha+\beta) / 2]}$ is a neighborhood of the compact set $\left|\Psi_{1}\right|_{\alpha} \cap\left|\Psi_{2}\right|_{\alpha}$ in $|\stackrel{\leftrightarrow}{\Psi}|_{\beta}$ so, by Lemma 3.1 the image of $\Pi_{k}\left(\left|\Psi_{1}\right|_{\alpha} \cap\left|\Psi_{2}\right|_{\alpha}\right)$ in $\Pi_{k}\left(\left|\mathscr{\Psi}_{1}\right|_{[(\alpha+\beta) / 2]} \cap\left|\Psi_{2}\right|_{[(\alpha+\beta) / 2]}\right)$ is finitely generated. Note that the generators can be assumed to be piecewise linear in 
$\left|\Psi_{2}\right|_{\beta}$. Since $\left|\Psi_{2}\right|_{\beta}$ is contractible each of these generators bounds a singular polyhedral cell. Corresponding to the groups

$$
\Pi_{1}\left(\left|\Psi_{1}\right|_{\alpha} \cap\left|\Psi_{2}\right|_{\alpha}\right), \Pi_{2}\left(\left|\Psi_{1}\right|_{\alpha} \cap\left|\Psi_{2}\right|_{\alpha}\right), \cdots, \Pi_{q-1}\left(\left|\Psi_{1}\right|_{\alpha} \cap\left|\Psi_{2}\right|_{\alpha}\right)
$$

and the groupoid $I_{0}\left(\left|\Psi_{1}\right|_{\alpha} \cap\left|\Psi_{2}\right|_{\alpha}\right)$ we get a finite collection of polyhedrai singular cells in $\left|\Psi_{2}\right|_{\beta}$ of dimension less than or equal to $q$. Let $P^{s}$ be the union of all these polyhedral singular cells. Using the topological engulfing we get a homeomorphism $g: M \rightarrow M$ such that $\left.g|| \Psi_{1}\right|_{[(\alpha+\beta) / 2]}=1$ and $P^{s} \subset g\left(\left|\Psi_{1}\right|_{\beta}\right)$. It is easy to see that

$$
i_{*}: \Pi_{r}\left(g\left(\left|\Psi_{1}\right|_{\alpha}\right) \cap\left|\Psi_{2}\right|_{\alpha}\right) \rightarrow \Pi_{r}\left(g\left(\left|\Psi_{1}\right|_{\beta}\right) \cap\left|\Psi_{2}\right|_{\beta}\right)
$$

is trivial for $r=0,1,2, \cdots, q-1$.

Using exactly the idea of the proof of the previous lemma, one can prove the following generalization.

Lemma 3.3. Let $M^{n}$ be a $q$-connected $n$-manifold $(q \leqq n-3)$, let $0<\alpha<\beta<1$ and let $\left|\Psi_{1}\right|, \cdots\left|\Psi_{m}\right|$ be n-cells in $M^{n}$. Then there exist homeomorphisms $g_{1}, \cdots, g_{m-1}$ of $M^{n}$ onto itself such that $\left.g_{i}|| \Psi_{i}\right|_{\alpha}=1$ and for any subset $k_{1}, k_{2}, \cdots, k_{r}$ of distinct integers between 1 and $m$ the map $i_{*}: I_{k}\left(\bigcap_{i=1}^{r}\left(\left|\Psi_{k_{i}}\right|_{\alpha}\right)\right) \rightarrow I I_{k}\left(\bigcap_{i=1}^{r} g_{k_{i}}\left(\left|\Psi_{k_{i}}\right|_{\beta}\right)\right.$ is trivial, where $i_{*}$ is induced by the inclusion map.

Proof of Theorem 1. Our proof is essentially a refinement of the proof of Lemma 2.3 interlacing the steps of the proof of 2.3 with the improvements of the intersection given by Lemma 3.3. Let $\left|\phi_{1}\right|,\left|\phi_{2}\right|,\left|\dot{\phi}_{3}\right|, \cdots$ be a collection of cells in $M^{n}$ such that $\bigcup_{i=1}^{\infty}\left|\phi_{i}\right|_{\alpha}$ covers $M^{n}$ for some $\alpha, 0<\alpha<1$. Let $\left(\alpha_{i}\right)$ and $\left(\beta_{i}\right)$ be sequences of real numbers such that $\alpha<\alpha_{1}<\beta_{1}<\alpha_{2}<\beta_{2}<\cdots$ and $\alpha_{i}<1$ for each $i$. As in the proof of Lemma 2.3 we stretch $\bigcup_{i=1}^{p}\left|\dot{\phi}_{i}\right|_{\alpha_{1}}$ over $\left|\dot{\phi}_{p+1}\right|_{\alpha}$ by $h_{1,1}, h_{2,1}, \cdots h_{p, 1}$ so that $\left.h_{i, 1}|| \phi_{i}\right|_{\alpha}=1$.

Next using Lemma 3.3 we get homeomorphisms $g_{1,1}, g_{2,1}, \cdots, g_{p, 1}$ of $M^{n}$ onto itself so that $\left.g_{i, 1}|| \phi_{i}\right|_{\alpha_{1}}=1$ and for any integers $k_{1}, k_{2}, \cdots, k_{r}$ between 1 and $p, i_{*}: \Pi_{k}\left(\bigcap_{i=1}^{r} h_{k_{\imath}}\left(\left|\phi_{k_{i}}\right|_{\alpha_{1}}\right)\right) \rightarrow I I_{k}\left(\bigcap_{i=1}^{r} g_{k_{i}{ }_{1}} h_{k_{i}, 1}\left(\left|\phi_{k_{1}}\right|_{\beta}\right)\right.$ is trivial for $0 \leqq k<q$. We continue this process first engulfing $\left|\phi_{p+2}\right|_{\alpha}$ then improving the intersections. For each $i=1,2, \cdots, p$ we get an increasing sequence of open cells $\left|\dot{\phi}_{i}\right|_{\alpha}, g_{i, 1} h_{i, 1}\left(\left|\dot{\phi}_{i}\right|_{\beta_{1}}\right), g_{1,2} h_{i, 2} g_{i, 1} h_{i, 1}\left(\left|\phi_{i}\right|_{\beta_{2}}\right)$, $\cdots$, whose direct limit is an open $n$-cell, call it $C_{i}$. For any collection $k_{1}, k_{2}, \cdots, k_{r}$ of integers between 1 and $p$ we see, using the fact that the groups of the intersection $\bigcap_{i=1}^{r} C_{k_{i}}$ are the direct limits of

$$
\bigcap_{i=1}^{r}\left|\phi_{k_{i}}\right|_{\alpha}, \bigcap_{i=1}^{r} g_{k_{i}} h_{k_{i}}\left(\left|\dot{\phi}_{k_{1}}\right|_{\beta_{1}}\right) \cdots \quad \text { that } \quad I I_{k}\left(\bigcap_{i=1}^{r} C_{k_{i}}\right)=0
$$

for $k=0,1,2, \cdots, q-1$. 
We should point out that a special case of Theorem 1 was proved by Zeeman in [6].

CoRollary 3.4. A contractible open $n$-manifold, $n \leqq 5$ is the union of two open cells whose intersection is a contractible open manifold.

Proof. By Theorem 1 we can cover such a manifold by two open cells $C_{1}$ ahd $C_{2}$ whose intersection is 1-connected. Using the MeyerVictoris sequence we get the exact sequence

$$
\cdots \rightarrow H_{k+1}\left(C_{1} \cup C_{2}\right) \rightarrow H_{k}\left(C_{1} \cap C_{2}\right) \rightarrow H_{k}\left(C_{1}\right) \oplus H_{k}\left(C_{2}\right) \rightarrow \cdots .
$$

But $H_{k+1}\left(C_{1} \cup C_{2}\right)=H_{k}\left(C_{1}\right)=H_{k}\left(C_{2}\right)=0$ for every $k$, so $H_{k}\left(C_{1} \cap C_{2}\right)=0$. The Hurewics isomorphism shows $\Pi_{k}\left(C_{1} \cap C_{2}\right)=0$ for each $k$. This implies that $C_{1} \cap C_{2}$ is contractible.

One might hope that the groups of the intersections of the covering cells might be improved to give trivial groups in dimension $q$. The following example shows that this may not be possible without using more cells to cover the manifold.

ExAMPLE. $S^{3} \times S^{3}$ can be covered by three open cells whose intersections are 1-connected. We show that these intersections cannot be improved to be 2-connected. Suppose, to the contrary that

$$
S^{3} \times S^{3}=C_{1} \cup C_{2} \cup C_{3}
$$

and that $C_{1} \cap C_{2}, C_{1} \cap C_{3}$ and $C_{2} \cap C_{3}$ are 2-connected. Using the Meyer-Victoris sequence we get $H_{3}\left(C_{1} \cup C_{2}\right) \oplus H_{3}\left(C_{2}\right) \rightarrow H_{3}\left(S^{3} \times S^{3}\right) \rightarrow$ $H_{2}\left(\left(C_{1} \cup C_{2}\right) \cap C_{3}\right)$ and $H_{3}\left(C_{1}\right) \oplus H_{3}\left(C_{2}\right) \rightarrow H_{3}\left(C_{1} \cup C_{2}\right) \rightarrow H_{2}\left(C_{1} \cap C_{2}\right)$ which implies $H_{3}\left(C_{1} \cup C_{2}\right)=0$. Furthermore we get the exact sequence

$$
H_{2}\left(C_{1} \cap C_{3}\right) \oplus H_{2}\left(C_{2} \cap C_{3}\right) \rightarrow H_{2}\left(\left(C_{1} \cup C_{2}\right) \cap C_{3}\right) \rightarrow H_{1}\left(C_{1} \cap C_{2} \cap C_{3}\right)
$$

but $H_{2}\left(C_{1} \cap C_{3}\right)=H_{2}\left(C_{2} \cap C_{3}\right)=0=H_{1}\left(C_{1} \cap C_{2} \cap C_{3}\right)$. This implies that $H_{2}\left(\left(C_{1} \cup C_{2}\right) \cap C_{3}\right)=0$. In the first given exact sequence we have all groups being trivial except $H_{3}\left(S^{3} \times S^{3}\right)$. This contradicts exactness. Note that if we are willing to use six open cells to cover $S^{3} \times S^{3}$ we can arrange it so that the intersections are open cells.

COROLLARY 3.5. Every topological n-manifold can be covered by $(n+1)$ open n-cells.

Corollary 3.6. If $M^{n}$ is a compact manifold $(n \leqq 5)$ that is a homotopy $n$-sphere then $M^{n}=S^{n}$.

Proof. $\quad M^{n}$ can be covered by two open cells hence $M^{n}=S^{n}$. 
4. Covering manifolds with boundary with closed cells. Using the methods of $\S 2$ we can get the following theorem.

THEOREM 4.1. If $M^{n}$ is a compact manifold with boundary and $M^{n}$ and $\mathrm{Bd} M^{n}$ are $q$ connected, $q \leqq n-4$ then $M^{n}$ can be covered by $p$ closed cells if $p(q+1)>n$.

We note that if $A^{n}$ is the closure of region between two tame $(n-1)$ spheres in $S^{n}$ then the annulus conjecture says that $A^{n}=$ $S^{n-1} \times[0,1]$. Note that the annulus conjecture is equivalent with the assertion that $A^{x}$ can be covered by two closed cells. It is the stumbling block presented by the annulus conjecture that prevents us from weakening the hypothesis of Theorem 4.1 to require only that each component of $\mathrm{Bd} M^{n}$ be $q$-connected.

5. An equivalence for the 3-dimensional Poincare conjecture. The Poincare conjecture says that a compact $n$-manifold without boundary that has the same homotopy groups as a sphere is a sphere. This conjecture is known to be true for $n \neq 3,4$. We prove the following:

THEOREM 4.1. The 3-dimensional Poincare conjecture is true if and only if every contractible open 3-manifold that is 1-connected at infinity is the union of two open cells.

Proof. According to Wall [5] if the Poincare conjecture is true, then an open 3-manifold that is 1-connected at infinity is a compact manifold minus a point. In the case of a contractible open manifold that is 1-connected at infinity, the 1-point compactification would be a homotopy 3-sphere, which again by the Poincare conjecture is a 3-sphere.

Conversely, suppose that each contractible open 3-manifold that is 1-connected at infinity is the union of two open cells. Let $S$ be a homotopy 3-sphere and $p \in S$. Then $M=S \sim\{p\}$ is a contractible open manifold that is 1-connected at infinity. By hypothesis $M=E_{1} \cup E_{2}$ where $E_{1}$ and $E_{2}$ are open 3-cells. Thus $S$ can be covered by three open cells. McMillan and Hempel [2] have shown that such a manifold is a 3-sphere with handles. But the only simply connected 3-sphere with handles is $S^{3}$.

\section{REFERENCES}

1. M. Brown, The monotone union of open $n$-cells is an open n-cell, Proc. Amer. Math. Soc. 12 (1961), 812-814.

2. J. Hempel and D. R. McMillan, Jr., Covering 3-manifolds with open cells. 
3. M. H. A. Newman, The engulfing theorem for topological manifolds, Ann. of Math. 84 (1966), 555-571.

4. J. L. Stern, Contractible open manifolds and cellular maps.

5. C. T. C. Wall, Open 3-manifolds which are 1-connected at infinity, Quart. J. Math 16 (1965), 263-268.

6. E. C. Zeeman, "The Poincare conjecture for $n \geqq 5$," topology of 3-manifolds and related topics, Prentice-Hall, Englewood Cliffs, N. J., 1963.

Received May 13, 1968.

UNIVERSITY OF IDAHO 



\section{PACIFIC JOURNAL OF MATHEMATICS}

\section{EDITORS}

H. ROYDEN

Stanford University

Stanford, California

Richard Pierce

University of Washington

Seattle, Washington 98105
J. DugundJI

Department of Mathematics

University of Southern California

Los Angeles, California 90007

BASIL GORDON

University of California

Los Angeles, California 90024

\section{ASSOCIATE EDITORS}

E. F. BECKENBACH

B. H. NeUmanN

F. WOLF

K. YOSHIDA

\section{SUPPORTING INSTITUTIONS}

UNIVERSITY OF BRITISH COLUMBIA

CALIFORNIA INSTITUTE OF TECHNOLOGY

UNIVERSITY OF CALIFORNIA

MONTANA STATE UNIVERSITY

UNIVERSITY OF NEVADA

NEW MEXICO STATE UNIVERSITY

OREGON STATE UNIVERSITY

UNIVERSITY OF OREGON

OSAKA UNIVERSITY

UNIVERSITY OF SOUTHERN CALIFORNIA
STANFORD UNIVERSITY

UNIVERSITY OF TOKYO

UNIVERSITY OF UTAH

WASHINGTON STATE UNIVERSITY

UNIVERSITY OF WASHINGTON

$* * \quad * \quad *$
AMERICAN MATHEMATICAL SOCIETY
CHEVRON RESEARCH CORPORATION
TRW SYSTEMS
NAVAL WEAPONS CENTER




\section{Pacific Journal of Mathematics}

Vol. 30, No. $1 \quad$ September, 1969

William Wells Adams, Simultaneous diophantine approximations and cubic irrationals ..................................... 1

Heinz Bauer and Herbert Stanley Bear, Jr., The part metric in convex

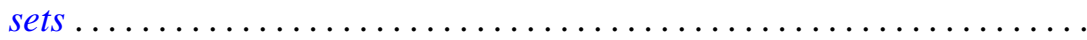

L. Carlitz, A note on exponential sums ...................... 35

Vasily Cateforis, On regular self-injective rings ................. 39

Franz Harpain and Maurice Sion, A representation theorem for measures on infinite dimensional spaces ......................... 47

Richard Earl Hodel, Sum theorems for topological spaces .............. 59

Carl Groos Jockusch, Jr. and Thomas Graham McLaughlin, Countable

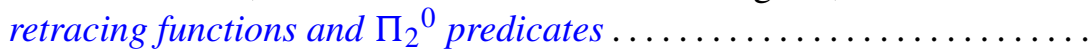

Bjarni Jónsson and George Stephen Monk, Representations of primary

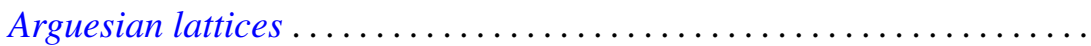

Virginia E. Walsh Knight, A continuous partial order for Peano continua...................................... 141

Kjeld Laursen, Ideal structure in generalized group algebras ........... 155

G. S. Monk, Desargues' law and the representation of primary lattices . . . 175

Hussain Sayid Nur, Singular perturbation of linear partial differential equation with constant coefficients ..........................

Richard Paul Osborne and J. L. Stern, Covering manifolds with cells ... 201

Keith Lowell Phillips and Mitchell Herbert Taibleson, Singular integrals in several variables over a local field...

James Reaves Smith, Local domains with topologically $T$-nilpotent radical....

Donald Platte Squier, Elliptic differential equations with discontinuous coefficients .................................

Tae-il Suh, Algebras formed by the Zorn vector matrix...

Earl J. Taft, Ideals in admissible algebras . .................... 259

Jun Tomiyama, On the tensor products of von Neumann algebras........ 263

David Bertram Wales, Uniqueness of the graph of a rank three group ..... 271

Charles Robert Warner and Robert James Whitley, A characterization of regular maximal ideals ......................... 